\title{
Images in Gene and Cell Therapy
}

\section{Direct Transfection of Dendritic Cells in the Epidermis After Plasmid Delivery Enhanced by Surface Electroporation}

\author{
Dinah H. Amante,, Trevor R.F. Smith,, Bill B. Kiosses, ${ }^{2}$ Niranjan Y. Sardesai, \\ Laurent M.P.F. Humeau, and Kate E. Broderick ${ }^{1}$
}

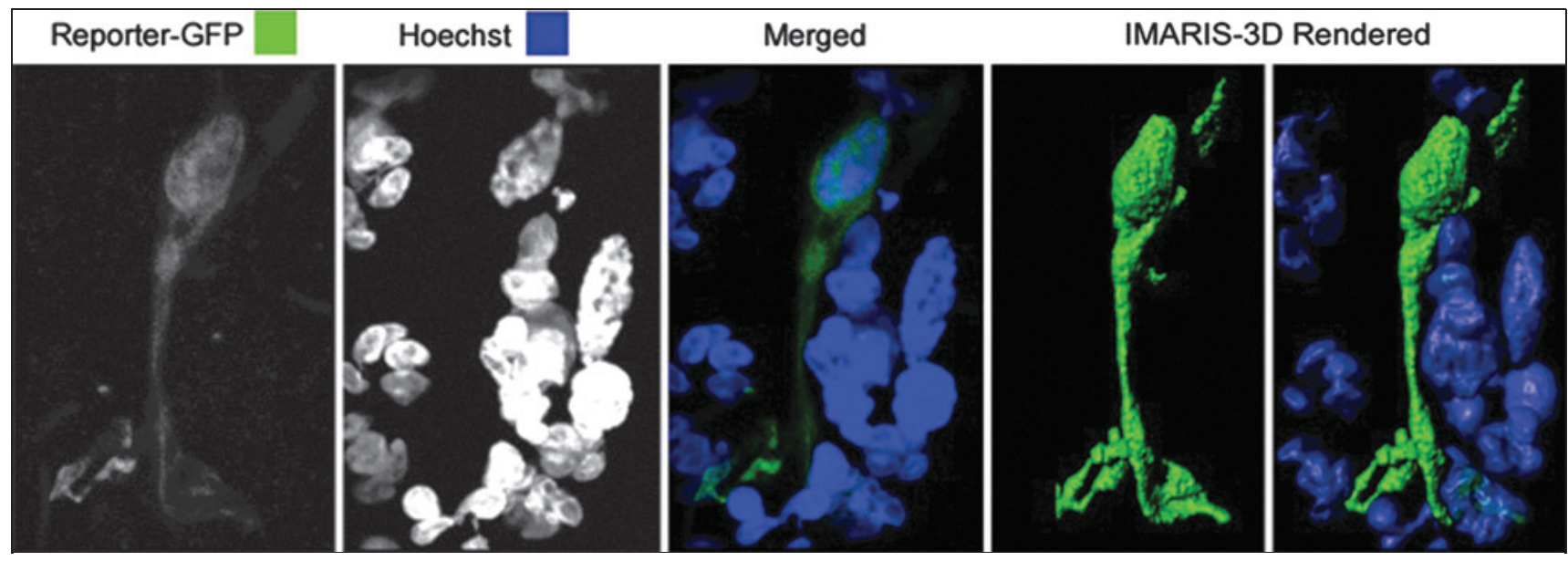

FIG. 1. Surface electroporation facilitates the direct transfection of dendritic cells in dermal tissue. Shown is a highpowered $(\times 63)$ confocal micrograph of a dendritic cell transfected with plasmid expressing GFP (green). The image is taken from a sectioned biopsy of guinea pig skin that had been treated with reporter plasmid, electroporated with the SEP device, and removed $6 \mathrm{hr}$ posttreatment. The skin section was processed with Hoechst stain (blue) to identify nuclei. The dendritic cell was captured in the dermal layer. IMARIS software was used postcapture to produce a three-dimensional image of the transfected cell and the nuclei of surrounding cells.

\section{Abstract}

The skin is rich in antigen-presenting cells and as such is an excellent target tissue for vaccination strategies. Electroporation is a physical delivery method that potentiates the uptake of DNA vaccines into target cells. Intradermal electroporation offers a minimally invasive solution to DNA delivery in the clinic. Here we describe the direct transfection of dendritic cells in the epidermis, using a surface dermal electroporation device, and specifically show a dendritic cell transfected with plasmid expressing green fluorescent protein. The dendritic cell has used its motile capabilities after transfection to move from the epidermis into the dermis, making its way to the lymphatic system.

$\mathbf{T}$ HE SKIN IS AN ATTRACTIVE TISSUE for vaccination in a clinical setting because of the ease of monitoring, the accessibility of the target, and most importantly the immunecompetent nature of dermal tissue. Although the main cell type in the epidermis is the keratinocyte, it is rich in dendritic cells including Langerhans cells, which account for $3-5 \%$ of all nucleated cells in the epidermis. This is in contrast to other tissues, such as muscle, that contain low numbers of professional antigen-presenting cells (Hohlfeld and Engel, 1994).

DNA vaccines may offer a solution to multiple pressing unmet health care needs as an immunotherapy against cancers and infectious diseases such as HIV/AIDS and hepatitis C (Sardesai and Weiner, 2011). However, naked delivery of DNA is not

\footnotetext{
${ }^{1}$ Inovio Pharmaceuticals, Plymouth Meeting, PA 19462.

${ }^{2}$ Core Microscopy Facility, Scripps Research Institute, La Jolla, CA 92037.
} 
sufficient to harness the full potential of the technology in large mammals or humans, with the paucity of in vivo transfection efficiency being cited as a limiting factor to the generation of robust immunogenicity (Weiner, 2008), and thus an enabling delivery technology is required. Although skin electroporation offers a novel methodology for the delivery of DNA vaccines in the clinic, little is known about the actual mechanism of the technique and the elucidation of the resulting immune responses.

Using a surface dermal electroporation (SEP) device (Broderick et al., 2011) that targets the epidermal layer of skin, we have previously demonstrated the direct transfection of keratinocytes by plasmid delivered intradermally (Mendoza et al., 2013). In addition, we also identified in the epidermis a dendritic cell population that was transfected (Smith et al., 2014). Here we describe an image of such a cell, previously identified by MsGp2 staining as a dendritic cell (Smith et al., 2014), which has been transfected with a DNA plasmid expressing the reporter gene encoding green fluorescent protein (GFP), using SEP device technology (Fig. 1). This image was captured $6 \mathrm{hr}$ posttreatment, and at that time point the cell was residing in the dermis. We infer that this cell has migrated out of the epidermis and is now interacting with nucleated nontransfected cells within the dermis. The device design, specifically related to electrode spacing, and the electrical parameters dictate that this cell could only have been transfected in the epidermis and therefore must possess motile properties allowing its transition into the dermal layer. The ability to directly transfect dendritic cells in the skin may have major implications for the nature, kinetics, and magnitude of the resulting immune response through this mode of delivery. Importantly, we have demonstrated that such cells can prime a host immune response (Smith et al., 2014).

Technically, the abdominal skin region of a female Hartley guinea pig was injected intradermally, using the Mantoux method, with $50 \mu \mathrm{l}$ of plasmid $(1 \mathrm{mg} / \mathrm{ml})$ GFP (gWIZ-GFP; Aldevron, Fargo, ND). Immediately after injection EP was performed by applying the SEP electrode array to the site. The animal was humanely killed $6 \mathrm{hr}$ later and the treatment site skin was excised, using an 8-mm biopsy punch. Skin biopsies were embedded in O.C.T. compound and sectioned at a thickness of $30 \mu \mathrm{m}$, using an OTF cryostat (Bright Instrument Co., Cambridge, UK) and Hoechst stained. Confocal images were obtained with a Zeiss LSM 780 laser scanning confocal microscope (Carl Zeiss, Jena, Germany) and processed with ZEN 2012 software (Carl Zeiss) and IMARIS-3D software (Bitplane, Zurich, Switzerland). Z stacks of images (obtained at 0.3$\mu \mathrm{m}$ intervals) were collected sequentially, using a $\times 63$ objective, followed by maximum intensity projection to create the single flattened stack presented in Fig. 1.

\section{Acknowledgments}

The authors thank Maria Yang and Lauren Jann for plasmid preparation and Katherine Schultheis for manuscript preparation assistance. This work was supported in part by a Department of Defense SBIR grant (phase I and phase II; no. W81XWH-11-C-0051).

\section{Author Disclosure Statement}

K.E.B., D.H.A., T.R.F.S., L.M.P.F.H., and N.Y.S. are current employees of Inovio and as such have financial interest (in the form of salary compensation, stock options, and/ or stock ownership) in the work described in this manuscript. B.B.K. is an Inovio collaborator who works at the Scripps Research Institute and has no potential conflicts of interest.

\section{References}

Broderick, K.E., Shen, X., Soderholm, J., et al. (2011). Prototype development and preclinical immunogenicity analysis of a novel minimally invasive electroporation device. Gene Ther. 18, 258-265.

Hohlfeld, R., and Engel, A.G. (1994). The immunobiology of muscle. Immunol. Today 15, 269-274.

Mendoza, J.M., Amante, D.H., Kichaev, G., et al. (2013). Elucidating the kinetics of expression and immune cell infiltration resulting from plasmid gene delivery enhanced by surface dermal electroporation. Vaccine 1, 384-397.

Sardesai, N.Y., and Weiner, D.B. (2011). Electroporation delivery of DNA vaccines: Prospects for success. Curr. Opin. Immunol. 23, 421-429.

Smith, T.R.F., Schultheis, K., Kiosses, W.B., et al. (2014). DNA vaccination enhanced by surface electroporation targets epidermal dendritic cells, initiating their rapid migration to the draining lymph node and induction of an accelerated immune response. Mol. Ther. Methods Clin. Dev. (In press). Weiner, D.B. (2008). DNA vaccines: Crossing a line in the sand. Introduction to special issue. Vaccine 26, 5073-5074.

Address correspondence to: Dr. Kate E. Broderick Inovio Pharmaceuticals 660 W. Germantown Pike, Suite 110 Plymouth Meeting, PA 19462

E-mail: kbroderick@inovio.com

Received for publication June 10, 2014; accepted after revision October 9, 2014.

Published online: October 21, 2014. 